\title{
Topological Frustration as a New Parameter to Tune Morphology Revealed Through Exploring the Continuum Between A-B-C 3-Arm Star and Linear Triblock Polymers.
}

Rohit Gupta,${ }^{\dagger}$ Mayank Misra, ${ }^{\dagger}$ Wenxu Zhang, ${ }^{\dagger}$ Ankita Mukhtyar, ${ }^{\ddagger}$ Samuel P. Gido, ${ }^{\dagger}$ Alexander Ribbe,$\uparrow$ Fernando A. Escobedo, $₫$ and E. Bryan Coughlin $\dagger^{\dagger *}$

$\dagger$ Department of Polymer Science \& Engineering, University of Massachusetts, 120 Governors

Drive, Amherst, Massachusetts 01003-4530, United States

School of Chemical and Biomolecular Engineering, Cornell University, 120 Olin Hall, Ithaca, New York 14853, United States

*Email: Coughlin@mail.pse.umass.edu (E.B.C.)

\section{Materials and methods}

\section{Materials}

Methyl Methacrylate (99\%, Alfa Aesar) and Styrene (99\%, Alfa Aesar) were passed through a column of basic alumina prior to use to remove the inhibiter. Azobisisobutyronitrile (AIBN) was recrystallized from methanol and stored at $0^{\circ} \mathrm{C}$ before use. Pentafluorophenyl maleimide ester (PFPMI) was synthesized as per the reported literature. ${ }^{1}$ Poly (ethylene glycol) methyl ether was purchased from Sigma Aldrich and was modified to amine-terminated Poly (ethylene glycol) using reported literature methods. ${ }^{2}$ Ruthenium(IV) oxide $0.5 \%$ in aqueous solution stabilized was purchased from Electron Microscopy Sciences.

\section{Instrumentation}

${ }^{1} \mathrm{H}$ NMR spectroscopy was performed on $500 \mathrm{MHz}$ Bruker 500 Ascend NMR spectrometer. Gel permeation chromatography (GPC) was performed in THF at a flow rate of $1.0 \mathrm{~mL} / \mathrm{min}$ using a refractive index detector on a Polymer Laboratories PL-GPC 50 Integrated GPC. Small-angle Xray scattering (SAXS) measurements were performed using a GANESHA 300 XL SAXS instrument. For SAXS measurements, the samples were prepared by dissolving the polymer in anhydrous benzene at a concentration of $50 \mathrm{mg} / \mathrm{mL}$ and were casted in $1 \mathrm{~mL}$ Teflon beakers. The beakers were covered with a glass hood to allow slow evaporation of the solvent at room temperature and the process was carried out for 5 days. After slow evaporation of the solvent at room temperature, the samples were vacuum dried overnight to remove any residual solvent in the film, followed by thermally annealing at $125^{\circ} \mathrm{C}$ for 5 days under vacuum. For transmission electron microscopy (TEM), thin polymer sections of approximately $40 \mathrm{~nm}$ in thickness were prepared by Leica (Reichert \& Jung) ULTRACUT Ultramicrotome using a diamond knife at room temperature. These sections were mounted on 400 mesh copper support grids and were stained using $\mathrm{RuO}_{4}$ for $10 \mathrm{~min}$. The stained thin sections were then examined on a JEOL 2000FX TEM operated at an accelerating voltage of $200 \mathrm{kV}$.

Zero Loss Energy Filtered Transmission Electron Microscopy (ZL-EFTEM) was performed on a JEOL JEM-2200FS EFTEM equipped with an Omaga-Type Electron Energy Loss Filter and a 
TVIPS TemCam-F416. To minimize electron-induced damage during observation all samples for EFTEM observations were cooled to $-177 \mathrm{degC}$ using a Gatan 636 Cryo-Holder. Total Electron doses were kept below $25 \mathrm{e}^{-2}$ (note: $50 \mathrm{e}^{-2}$ is about the max dose acceptable for vitrified sample) with acquisition times between 5-10 seconds. While one can never exclude that especially the PMMA experiences some decay during e-beam exposure we did not observe change of the recorded morphology during the respective exposure times. To maximize contrast both a highcontrast objective aperture, which helps to increase elastic scattering contrast, and a $10 \mathrm{eV}$ postfilter slit aperture, which provides contrast enhancement due to the blocking of inelastically scattered electrons, were employed. ${ }^{3}$ Image post-processing was performed applying 2D-FFT filtering by removing high-frequency noise through the application of a circular mask with a size slightly larger than the respective domain spacings measured by SAXS i.e. all frequencies smaller than the measured expected domain spacings are removed.

\section{Dissipative Particle Dynamics}

Due to the length scales associated with the relevant morphologies, we employed Dissipative Particle Dynamics (DPD) to investigate the effect of change in grafting position in a coarse-grained triblock polymer model. DPD is an off-lattice mesoscopic molecular dynamics technique that bridges the gap between microscopic and macroscopic simulations. The DPD particles, representing a Gaussian-like chain, interact by conservative $\left(F^{C}\right)$, dissipative $\left(F^{D}\right)$, random $\left(F^{R}\right)$, and bond $\left(F^{B}\right)$ forces which are pairwise additive. The conservative force is the primary driving force towards equilibrium, while the dissipative and random forces act as a thermostat and dynamic tuning parameters. The forces on an $i^{\text {th }}$ particle is only exerted by particles within a cut-off radius, $r_{c}$. The momentum-conserving forces are given by $F_{i j}^{D}=-\gamma\left(1-r_{i j} / r_{c}\right)^{2}\left(\hat{r}_{i j} \cdot \vec{v}_{i j}\right)$ and $F_{i j}^{R}=$ $\sqrt{2 \gamma k_{B} T}\left(1-r_{i j} / r_{c}\right) \hat{r}_{i j}(\Delta t)^{-1 / 2}$, where $\gamma$ is the friction coefficient, $\Delta t$ is the integrating time step, $k_{B}$ is the Boltzmann constant and $T$ is the temperature. The units are scaled such that $r_{c}=k_{B} T=1$ and $\Delta t=0.04$. The soft repulsive conservative component is given by: $F_{i j}^{C}=a_{i j}\left(1-r_{i j} / r_{c}\right) \hat{r}_{i j}$, where $r_{i j}=r_{i}-r_{j}$ and $\hat{r}_{i j}=r_{i j} /\left|r_{i j}\right|$.

\section{Synthesis of RAFT Chain Transfer Agent for MMA Polymerization.}

The synthesis of Reversible Addition Fragmentation Transfer (RAFT) chain transfer agent (CTA1a) was performed following a reported procedure. ${ }^{4}$ CTA-1a was modified to CTA-1b using dicyclohexylcarbodimide-4-N,N dimethaminopyridine (DCC-DMAP) coupling reaction. In a round bottom flask, CTA-1a $(2.55 \mathrm{~g}, 8.75 \mathrm{mmol})$ and ethanol $(1.1 \mathrm{~mL}, 17.5 \mathrm{mmol})$ were added to $30 \mathrm{~mL}$ dichloromethane (DCM) and stirred at room temperature for 2 days (Scheme 1). The product obtained was purified by column chromatography using Hexane: EtOAc $=9: 1$ as the eluting solvent mixture to afford the desired product in $95 \%$ yield.

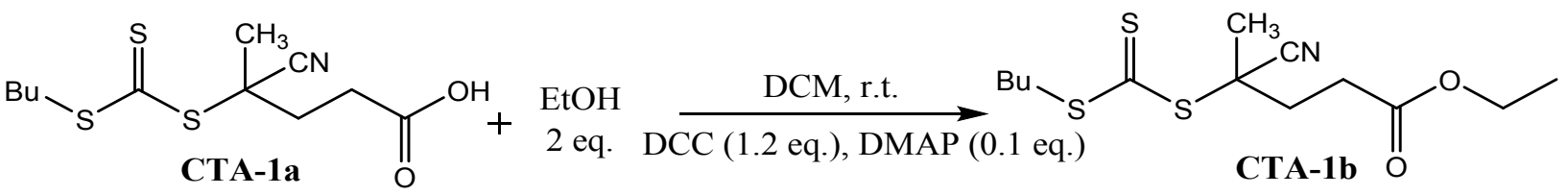


Scheme 1: DCC-DMAP coupling reaction for modification of CTA-1a to CTA-1b.

\section{General Procedure for Polymerization of MMA using CTA-1b.}

Methyl methacrylate $(20 \mathrm{~mL}, 0.19 \mathrm{~mol})$ was added to a Schlenk tube containing CTA-1b and AIBN as the initiator in appropriate quantities based on the targeted molecular weight (Table 1). The polymerization was performed using a molar ratio of AIBN/CTA at 1:20. The mixture was degassed by 3 freeze-pump-thaw cycles and then heated at $60{ }^{\circ} \mathrm{C}$ for different time intervals to achieve the required conversion. On completion, the reaction was quenched in an ice bath, diluted with ethyl acetate, and precipitated in methanol. The precipitated polymer was filtered and dried overnight under vacuum to yield PMMA macro CTA as a white powder.

\section{General Procedure for Chain Extension using Styrene (PMMA-b-PS 1 )}

The chain extension of styrene was performed using the synthesized PMMA macro CTA as the suitable RAFT agent and AIBN as the initiator. In a $20-\mathrm{mL}$ reaction vial, freshly distilled styrene, AIBN, and PMMA macro CTA in 1,4 dioxane were added. The different molar ratio of styrene:macro-CTA were taken based on the targeted molecular weights and the AIBN:macroCTA ratio of 1:20 was kept constant. The reaction mixture and was degassed using $\mathrm{N}_{2}$, followed by polymerization at $60{ }^{\circ} \mathrm{C}$ for different time intervals to achieve the desired conversion. After completion, the reaction mixture was quenched in an ice bath, diluted with ethyl acetate, and then precipitated in methanol. The precipitated polymer was filtered and dried overnight under vacuum to give PMMA- $b-\mathrm{PS}_{1}$ as a white powder. The diblock polymer was further purified to remove the PMMA homopolymer contamination by washing the PMMA- $b-\mathrm{PS}_{1}$ using $\mathrm{CH}_{3} \mathrm{NO}_{2}$ : $\mathrm{MeOH}(3: 7)$ solution mixture.

\section{General Procedure for Single-Molecule Insertion (SMI) of Pentafluorophenyl Maleimide (PFPMI).}

In a 20-mL reaction vial, PMMA macroinitiator (1 equiv.) dissolved with an appropriate amount of 1,4 dioxane was added. PFPMI ( 3 equiv.) and AIBN ( 0.1 equiv.) were added. The reaction vial was sealed and degassed using $\mathrm{N}_{2}$ and immersed in a preheated oil bath heated at $60{ }^{\circ} \mathrm{C}$ for 3 days to ensure completion insertion of the PFPMI molecule on the PS chain-end. The reaction mixture was diluted using ethyl acetate and precipitated in methanol. The precipitated polymer was filtered and dried under vacuum for $24 \mathrm{~h}$ to obtain PMMA-PFPMI as a white solid. Following a similar procedure, PMMA- $b$-PS 1 -PFPMI was synthesized by performing single-molecule insertion using the PMMA- $b-\mathrm{PS}_{1}$ macroinitiator.

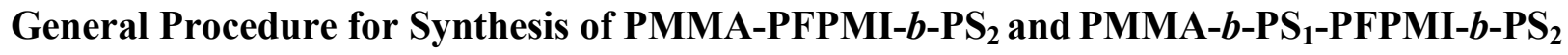

\section{Block polymers.}

In a $20-\mathrm{mL}$ reaction vial, PMMA-PFPMI macroinitiator, freshly distilled styrene, and AIBN dissolved in 1, 4 dioxane were added. The amount of styrene and AIBN was calculated based on the molar ratio of styrene:macro-CTA and AIBN:macro-CTA (20:1) respectively. The reaction 
vial was sealed, and the polymerization was performed at $60{ }^{\circ} \mathrm{C}$ for a specific time interval to achieve the targeted conversion. The reaction mixture was diluted using ethyl acetate upon completion and precipitated in methanol three times. The precipitated polymer was then filtered and dried under vacuum to yield PMMA-PFPMI- $b-\mathrm{PS}_{2}$ as a white solid. Similarly, PMMA- $b-\mathrm{PS}_{1}$ PFPMI- $b-\mathrm{PS}_{2}$ was synthesized using PMMA- $b-\mathrm{PS}_{1}$-PFPMI as the macro CTA for performing the styrene chain extension. These synthesized diblock polymers were further purified by successful removal of PS homopolymer using THF: Hexane (1:3) solution mixture, generated during the two styrenic chain extensions.

\section{General Procedure for Grafting of Amine-Terminated PEO on PMMA-PFPMI- $b-$ PS $_{2}$, PMMA-b-PS 1 -PFPMI- $b$-PS ${ }_{2}$, and PMMA-b-PS 1 -PFPMI}

The synthesized diblock polymer PMMA-PFPMI- $b-\mathrm{PS}_{2}$ was dissolved in chloroform in a $20-\mathrm{mL}$ reaction vial and amine-terminated PEO (1.1 equiv.) was added. $\mathrm{NEt}_{3}(0.1$ equiv.) was added and the vial was sealed and placed in a preheated oil bath at $60{ }^{\circ} \mathrm{C}$ for 3 days. On completion, the reaction mixture was diluted with ethyl acetate, precipitated in cold diethyl ether, and dried under vacuum for $24 \mathrm{~h}$. To remove the excess $\mathrm{PEO}$, the dried polymer was washed with $\mathrm{H}_{2} \mathrm{O}$ : $\mathrm{MeOH}$ (9:1) solution mixture to yield PMMA- $b$-PS- $g$-PEO star polymer architectures. Following a similar procedure, amine-terminated PEO was grafted onto PMMA- $b$-PS 1 -PFPMI- $b-\mathrm{PS}_{2}$ and PMMA- $b$ $\mathrm{PS}_{1}$-PFPMI to yield PMMA- $b$-PS-g-PEO graft and linear triblock polymer architectures respectively.

Table S1. Synthesis of PMMA macro-initiator using CTA-1b

\begin{tabular}{|c|c|c|c|c|}
\hline Sample & $\begin{array}{c}{[\mathrm{CTA}]} \\
(\mathrm{mmol} / \mathrm{L})\end{array}$ & $\begin{array}{c}{[\mathrm{AIBN}]} \\
(\mathrm{mmol} / \mathrm{L})\end{array}$ & $\begin{array}{c}\mathrm{M}_{\mathrm{n}, \mathrm{GPC}} \\
(\mathrm{kg} / \mathrm{mol})\end{array}$ & Đ \\
\hline PMMA- 30K & 0.31 & 0.016 & 30.2 & 1.12 \\
\hline PMMA- 25K & 0.21 & 0.011 & 25.6 & 1.14 \\
\hline
\end{tabular}




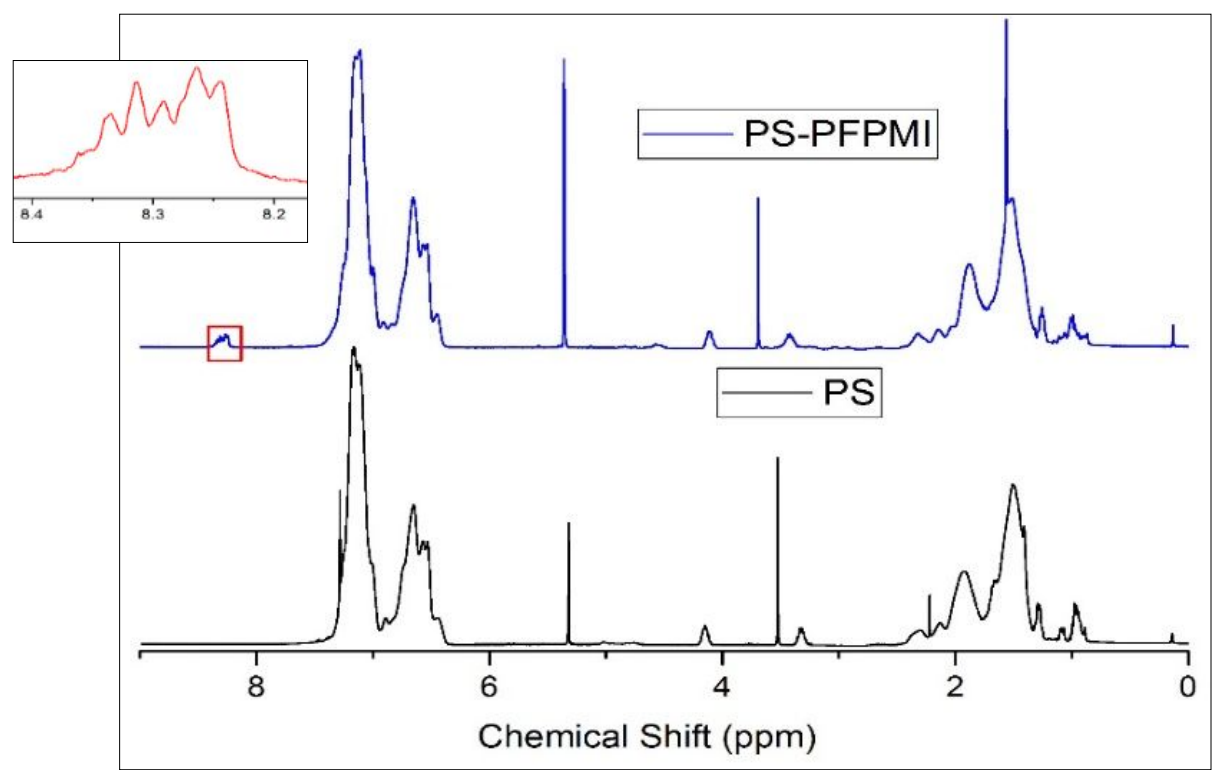

Figure S1: ${ }^{1} \mathrm{H}$ NMR data for SMI of PFPMI molecule, inset is the aromatic region for PFPMI molecule (top) on PS chain-end (bottom).

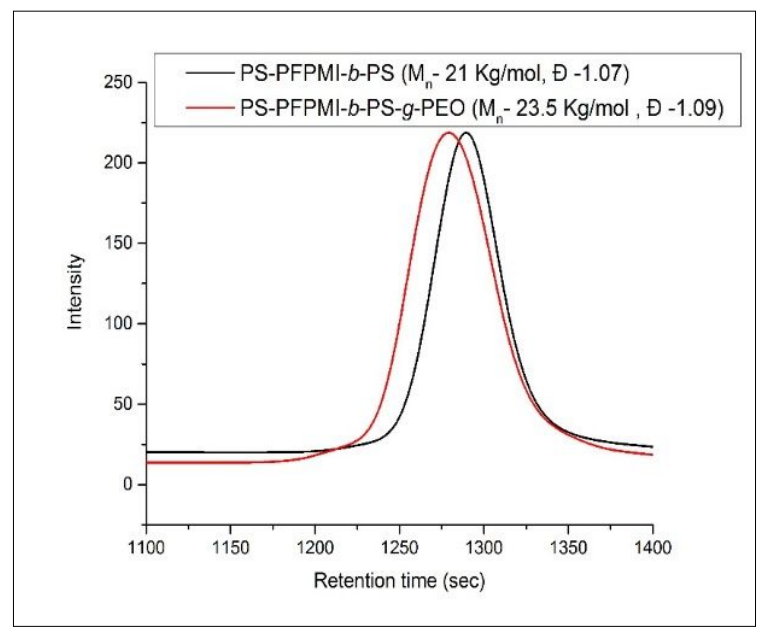

Figure S2: GPC traces demonstrating successful grafting of PEO on to PS-PFPMI-PS to obtain PS-PFPMI-PS- $g$-PEO graft polymer. 


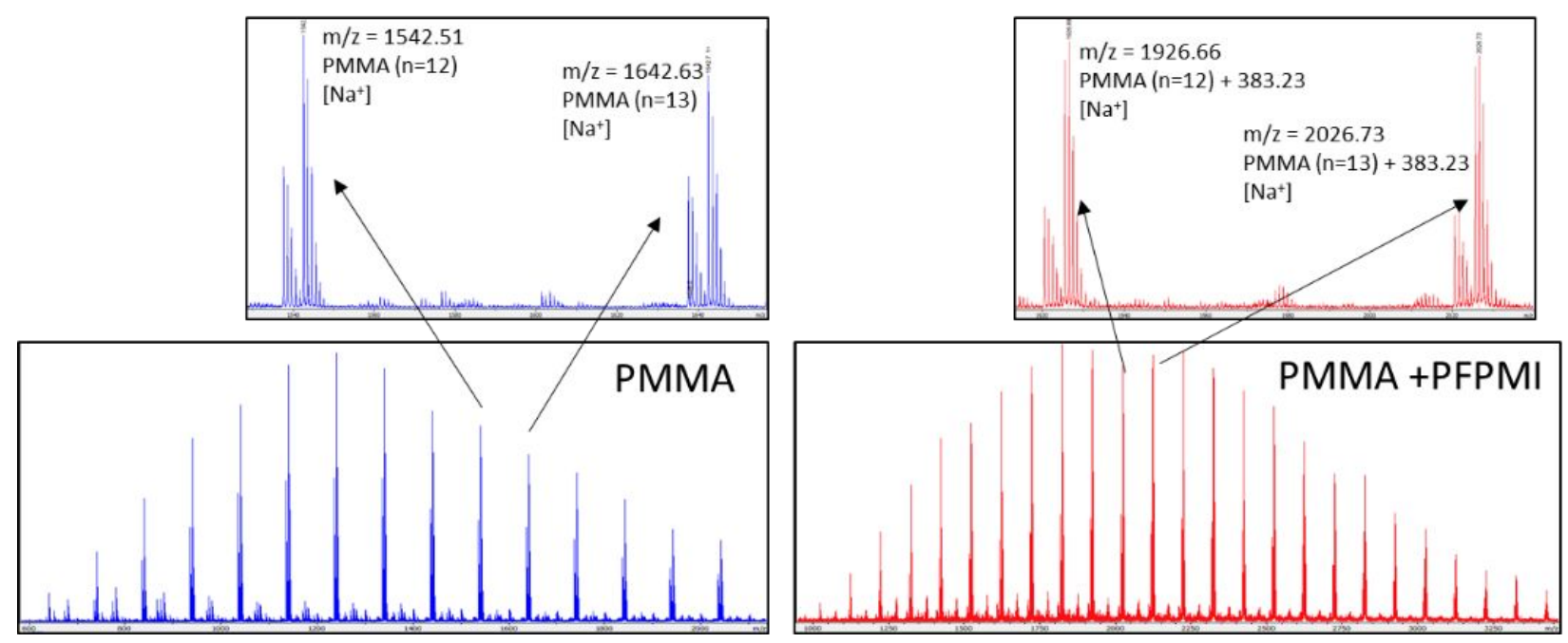

Figure S3: Molecular weight distribution using ESI-MS for PMMA (left) and PMMA-PFPMI (right).

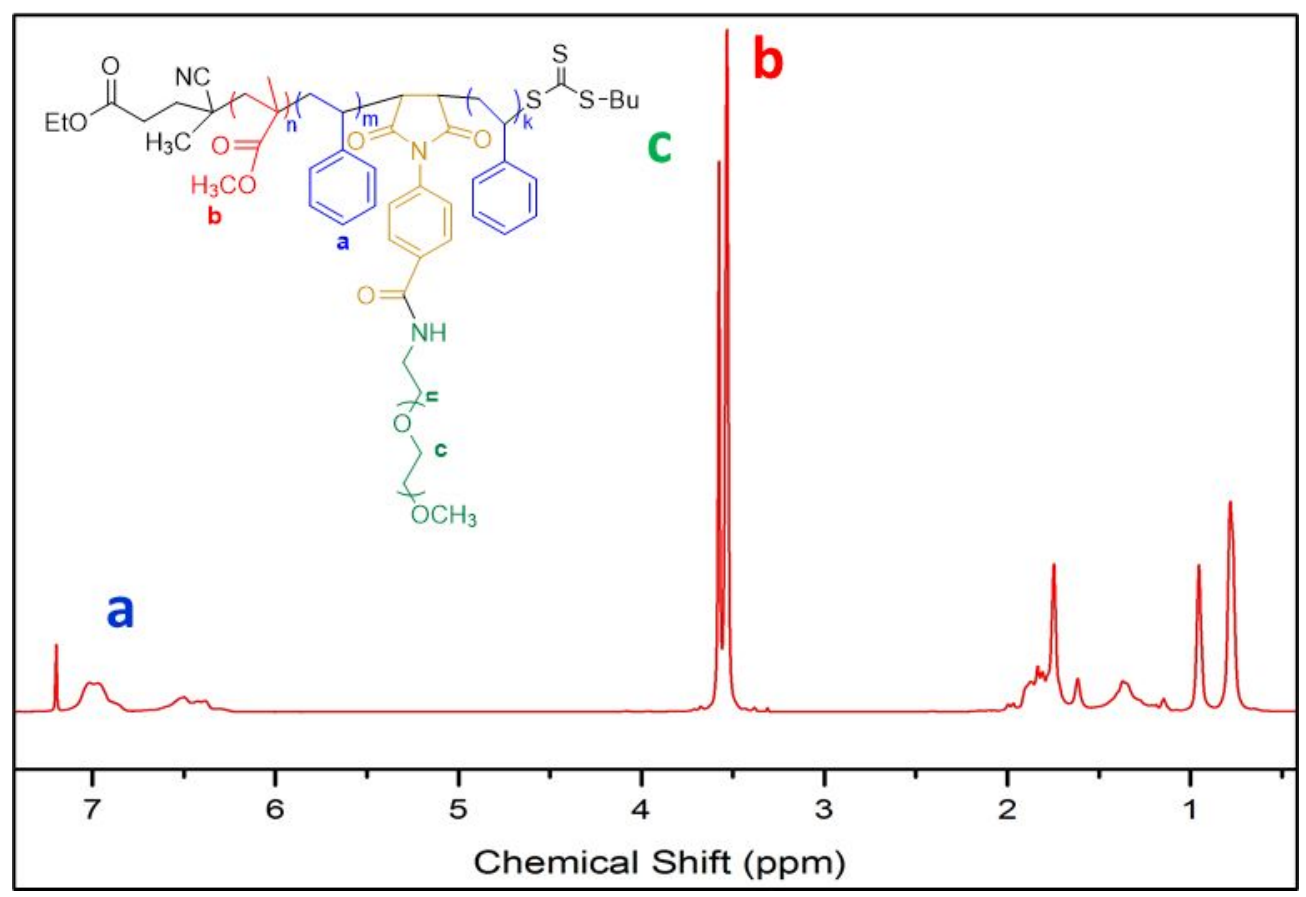

Figure S4: ${ }^{1} \mathrm{H}$ NMR data for synthesized PMMA- $b$-PS ${ }_{1}$-PFPMI- $b-\mathrm{PS}_{2}-g$-PEO continuum graft polymer. 


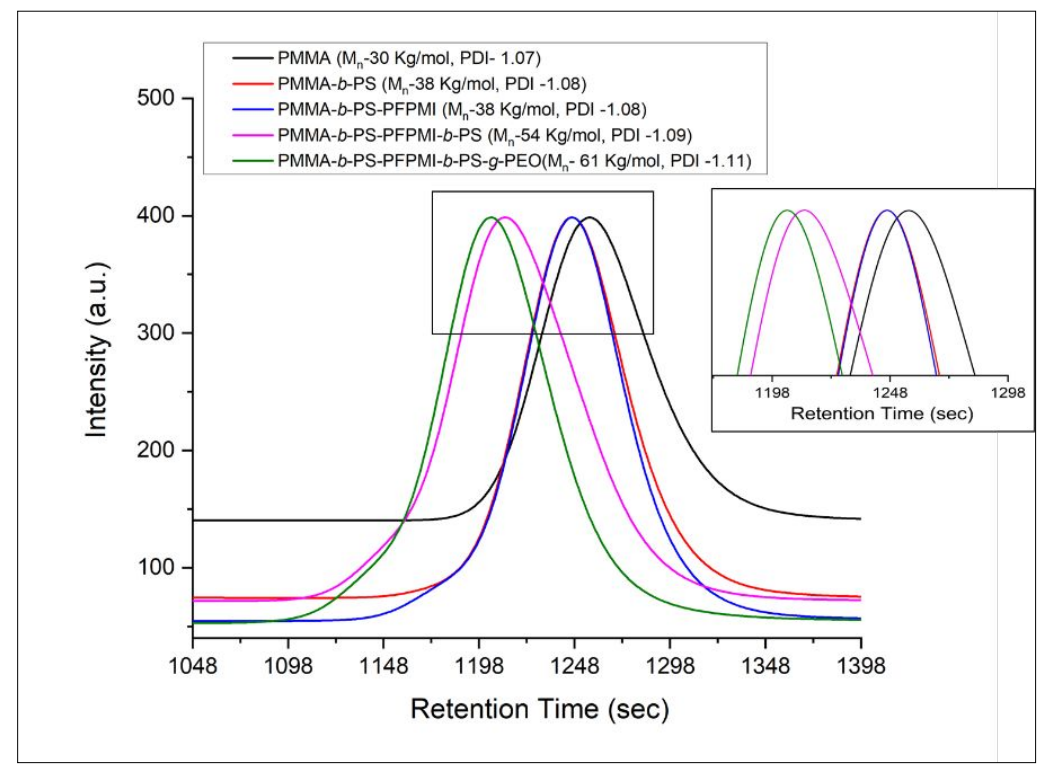

Figure S5: GPC traces for different steps in the synthesis of PMMA- $b$-PS ${ }_{1}-\mathrm{PFPMI}-b-\mathrm{PS}_{2}-g$-PEO, continuum graft polymers with $\omega=1 / 3$.

Table S2. Compositions for the 25-8- $\omega$, and 30-18- $\omega$ Series.

\begin{tabular}{|c|c|c|c|c|c|}
\hline Sample ID & $\begin{array}{c}\text { PMMA } \\
\text { (Kg/mol) }\end{array}$ & $\begin{array}{c}\mathrm{PS}_{1} \\
(\mathrm{Kg} / \mathrm{mol})\end{array}$ & $\begin{array}{c}\mathrm{PS}_{2} \\
(\mathrm{Kg} / \mathrm{mol})\end{array}$ & $\begin{array}{c}\text { PEO } \\
\text { (Kg/mol) }\end{array}$ & $\omega$ \\
\hline $25-8-0$ & 25 & 0 & 25 & 8 & 0 \\
\hline $25-8-(1 / 3)$ & 25 & 8 & 16 & 8 & $1 / 3$ \\
\hline $25-8-(2 / 3)$ & 25 & 16 & 8 & 8 & $2 / 3$ \\
\hline $25-8-(1)$ & 25 & 25 & 0 & 8 & 1 \\
\hline $30-18-(0)$ & 30 & 0 & 30 & 18 & 0 \\
\hline $30-18-(1 / 3)$ & 30 & 10 & 20 & 18 & $1 / 3$ \\
\hline $30-18-(2 / 3)$ & 30 & 20 & 10 & 18 & $2 / 3$ \\
\hline $30-18-(1)$ & 30 & 30 & 0 & 18 & 1 \\
\hline
\end{tabular}

Table S3: Estimated contrast for stained and unstained TEM images for different mixed domains observed for continuum graft polymers.

\begin{tabular}{|c|c|c|c|c|}
\hline \multirow[t]{2}{*}{ Sample } & \multicolumn{4}{|c|}{ Intensity } \\
\hline & Bright & & & Dark \\
\hline Stained & PMMA & PMMA-PEO/PMMA & PEO-PS/PS & PEO \\
\hline
\end{tabular}




\begin{tabular}{|l|lllc|}
\hline Unstained & PS & PMMA-PEO/PEO & PEO-PS/PMMA & PEO \\
\hline
\end{tabular}

Heavy metal staining is commonly used to identify morphologies on multi-phase polymer systems, and it is probably fair to say that Osmiumtertaoxide $\left(\mathrm{OsO}_{4}\right)$ and Rutheniumtetraoxide $\left(\mathrm{RuO}_{4}\right)$ are the most wildly applied staining agents. While $\mathrm{OsO}_{4}$ is well known to react specifically with double bonds ${ }^{5} \mathrm{RuO}_{4}$ is relatively unspecific and will stain various polymers including PEO and PS depending on the staining conditions, while it leaves PMMA untouched. ${ }^{6}$. In the case of a PS/PMMA blend $\mathrm{RuO}_{4}$ stains the PS phase. ${ }^{7}$ It has been shown that there is an impact on domain spacing $^{8}$ or susceptibility to staining artifacts ${ }^{9}$ in PS. In a PS-b-PEO diblock polymer, the PEO is stained quicker than the PS under moderate staining conditions. ${ }^{10}$ Relatively little is known about the chemical reactions the staining agent undergoes with the various polymer systems, but it is well established that $\mathrm{RuO}_{4}$ tends to accumulate in the lower density phases such as amorphous regions. ${ }^{11,12}$

The staining procedure applied for the here for the presented samples should mostly stain the PEO phase, which will, therefore, control the contrast observed on the bright-field TEM images. We, however, cannot exclude the possibility that some staining of the PS phase might occur. Assuming the PS is lightly stained with $\mathrm{RuO}_{4}$, and PMMA being probably decayed due to beam damage will appear brighter. In comparison, the darkest phase in both bright-field TEM and ZL-EFTEM should be PEO, while the contrast of PMMA and PS will be inverted.

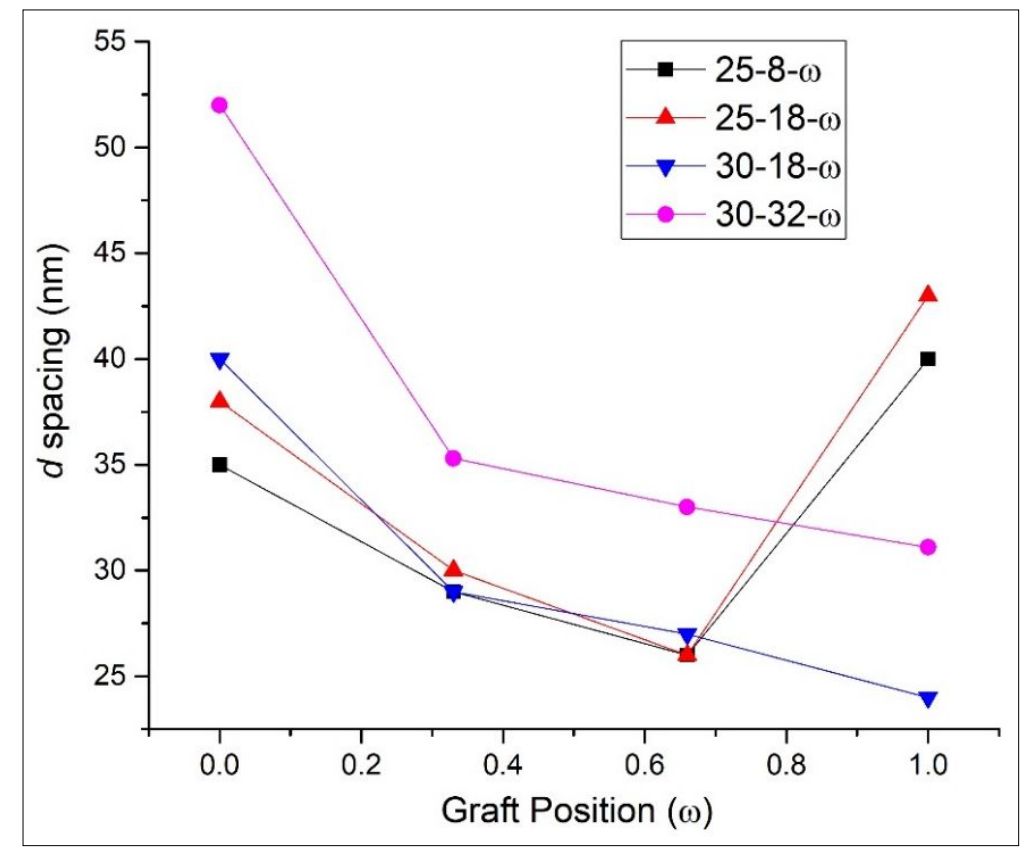

Figure S6: Effect of graft position on domain spacing for $25-\mathrm{Y}-\omega$ and $30-\mathrm{Y}-\omega$ series. 

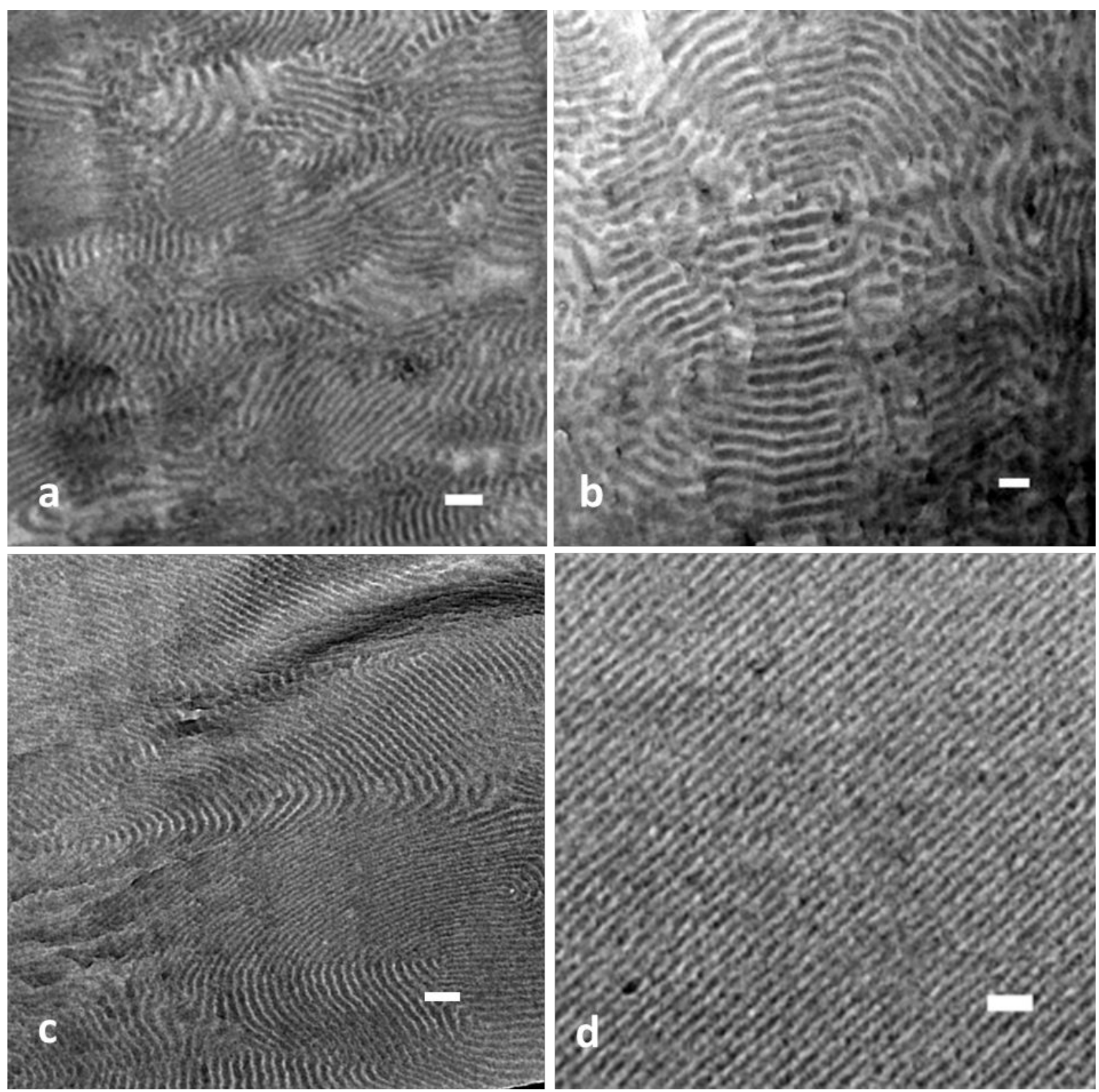

Figure S7. TEM micrographs for different continuum block polymers with varying $\omega$ values. (ad) $25-8-\omega, \omega=0,1 / 3,2 / 3,1$, respectively. Scale bar: $100 \mathrm{~nm}$. 

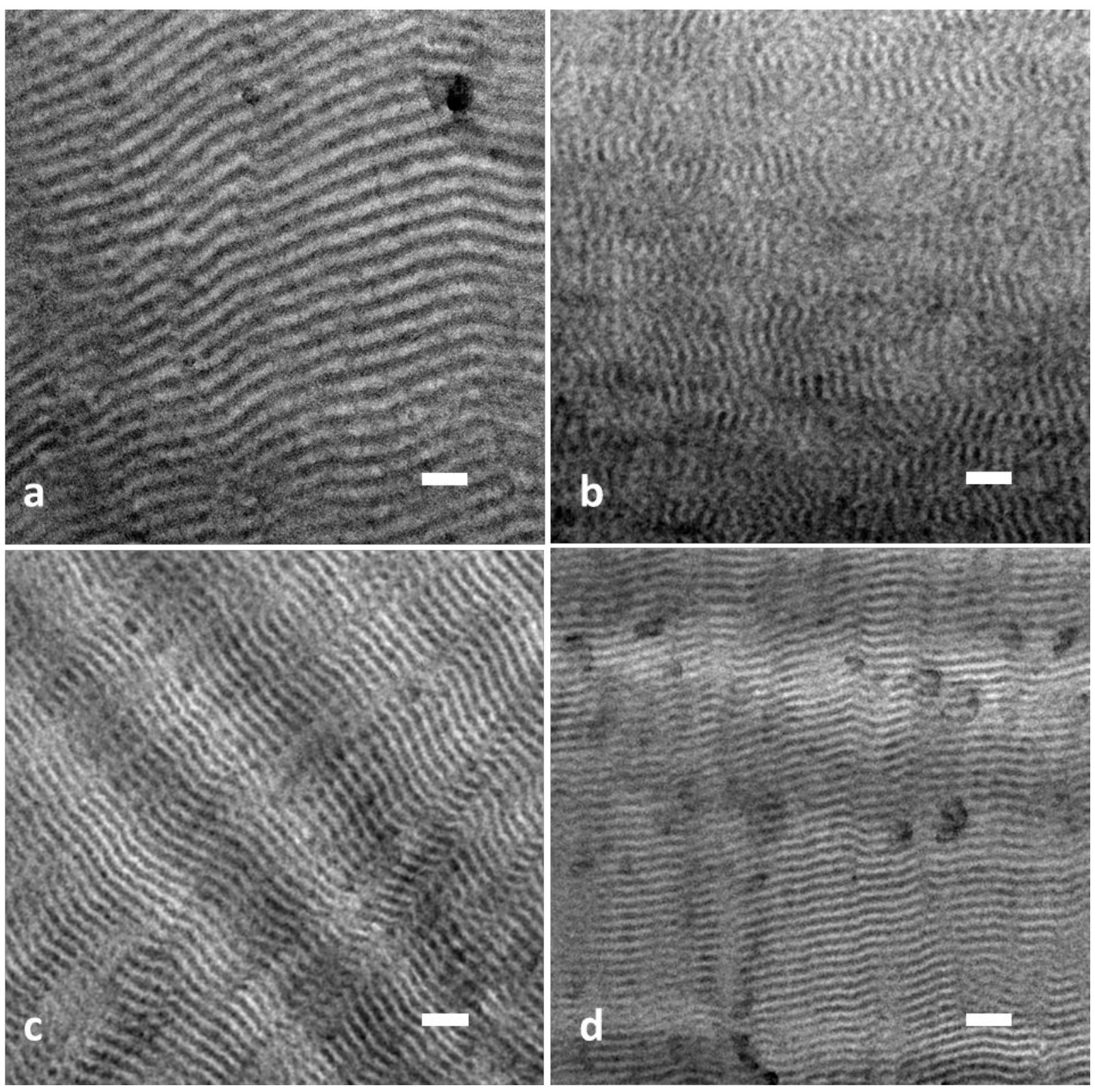

Figure S8. TEM micrographs for different continuum block polymers with varying $\omega$ values. (ad) 30-18- $\omega, \omega=0,1 / 3,2 / 3,1$, respectively. Scale bar: $100 \mathrm{~nm}$. 

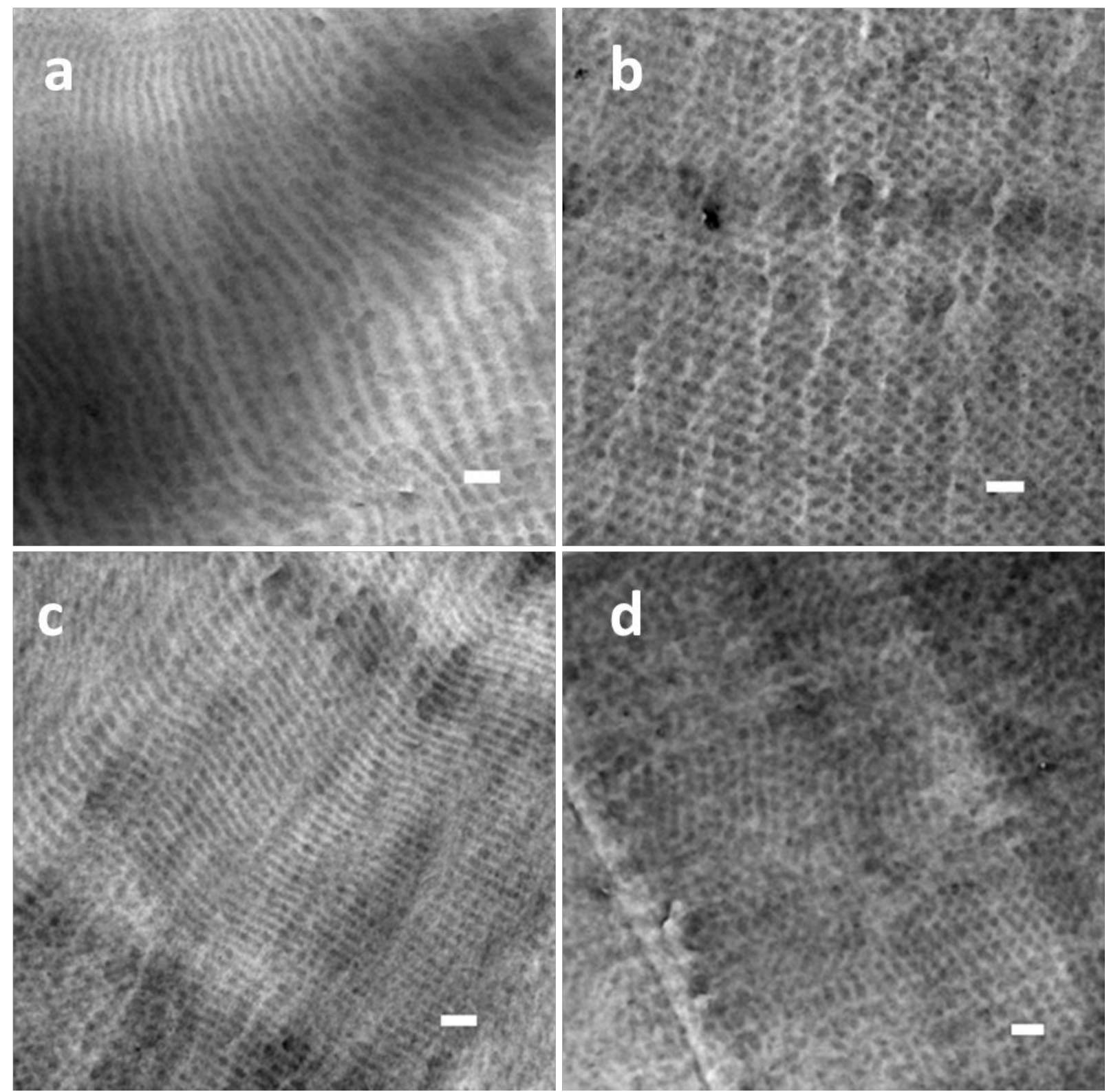

Figure S9: TEM micrographs for different continuum graft polymers with varying $\omega$ values (a-d) 30-32- $\omega, \omega=0,1 / 3,2 / 3,1$, respectively Scale bar: $100 \mathrm{~nm}$.

The average fraction of PMMA beads interacting with PEO beads is shown in Figure 7. To calculate the fraction of PMMA beads interacting with PEO beads, we draw a sphere of radius $\mathrm{R}_{\mathrm{c}}$ $\left(\mathrm{R}_{\mathrm{c}}=1\right)$ around a PMMA bead and check if any PEO bead lies inside its interaction field. If so, then the PMMA bead is marked as interacting. We calculate the total number of PMMA beads marked as interacting and normalize it by the total number of PMMA beads in the system to get the fraction of PMMA beads interacting with PEO. The fraction is calculated for each frame of the simulation and results averaged over all frames to get the final value. The error bars are associated with the standard deviation over the data set. 


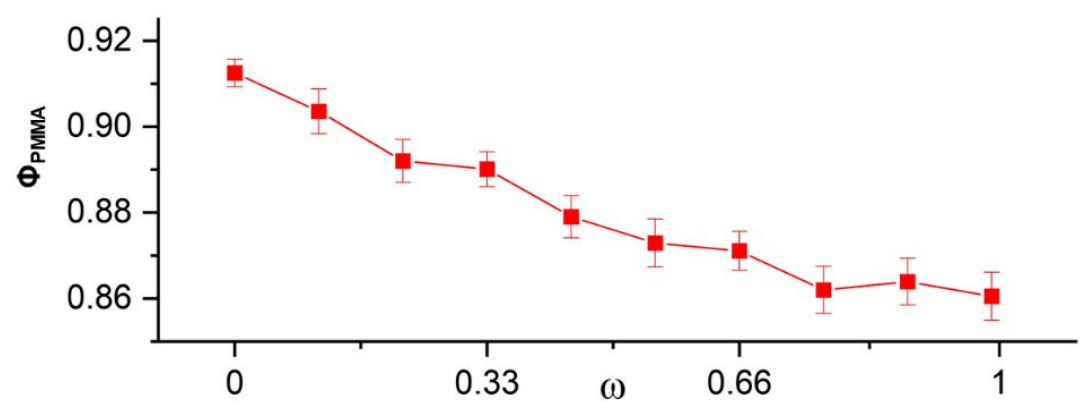

Figure S10: Fraction of PMMA beads $\left(\Phi_{\text {PMMA }}\right)$ interacting with PEO predicted using DPD simulations.

\section{References:}

1. Kakuchi, R., Zamfir, M., Lutz, J. F. \& Theato, P. Controlled positioning of activated ester moieties on well-defined linear polymer chains. Macromolecular Rapid Communications 33, 54-60 (2012).

2. Mahou, R. \& Wandrey, C. Versatile route to synthesize heterobifunctional poly(ethylene glycol) of variable functionality for subsequent pegylation. Polymers 4, 561-589 (2012).

3. Libera, M. R. \& Egerton, R. F. Advances in the transmission electron microscopy of polymers. Polymer Reviews 50, 321-339 (2010).

4. Moad, G., Chong, Y. K., Postma, A., Rizzardo, E. \& Thang, S. H. Advances in RAFT polymerization: The synthesis of polymers with defined end-groups. Polymer 46, 84588468 (2005).

5. Ribbe, A.E.; Bodycomb, J.; Hashimoto, T. Quantitative Analysis of the Staining of a Polyisoprene-block-polystyrene. Macromolecules 32, 3154-3156 (1997).

6. Trent, J.S.; Scheinbeim, J.I.; Couchman, P.R. Ruthenium tetraoxide staining of polymers for electron microscopy. Macromolecules 16, 589-598 (1983).

7. Trent, J.S.; Scheinbeim, J.I.; Couchman, P.R. Ruthenium Electron microscopy of PS/PMMA and rubber-modified polymer blends: Use of ruthenium tetroxide as a new staining agent. Journal of Polymer Science: Polymer Letters Edition 19, 315-319 (1981).

8. Park S.-M.; Yun, S.-H.; Sohn, B.-H. Variations of the lamellar period in thin films of diblock copolymers by selective staining agents. Macromolecular Chemistry and Physics 203, 2069-2074 (2002).

9. Chou, T.M.; Prayoonthong, P.; Aitouchen, A.; Libera, A. Nanoscale artifacts in $\mathrm{RuO}_{4}$ stained poly(styrene) $\mathrm{RuO}_{4}$ staining artifact of PS. Polymer 43, 2085-2088 (2002).

10. Zhu, L.; Huang, P.; Cheng, S.C.S; Ge, Q.; Quirk, R.P.; Thomas, E.L.; Lotz, B.; Wittmann, J.-C.; Hsiao, B.S.; Yeh, F.; Liu, L. Dislocation-Controlled Perforated Layer Phase in a PEO--PS Diblock Copolymer. Phys. Rev. Lett. 86, 6030 (2001). 
11. Foks, J.; Janik, H.; Pohl, M. Ruthenium tetroxide staining of semicrystalline polyester polyurethane films. European Polymer Journal 27, 729-733 (1991).

12. Li, J.X.; Cheung, W.L. Exposing lamellar structures in PP. Applied Polymer 72, 15291538 (1999) 